\title{
Prepuce-sparing corporoplasty as a safe alternative for patients with acquired penile curvature
}

\author{
Aldo Franco De Rose ${ }^{1}$, Francesca Ambrosini ${ }^{1}$, Guglielmo Mantica ${ }^{1}$, Enrico Zero ${ }^{2}$, \\ Riccardo Banchero ${ }^{1}$, Carlo Terrone ${ }^{1}$ \\ ${ }^{1}$ Department of Urology, Policlinico San Martino Hospital, University of Genoa, Genoa, Italy; \\ 2 Department of Computer Science, Bioengineering, Robotics and Systems Engineering, University of Genoa, Genoa, Italy.
}

\begin{abstract}
Summary Objectives: penile curvature is a rare condition, classified as congenital or acquired

(Peyronie's disease) (PD). Surgical correction is the standard treatment. It's common practice to associate circumcision with penile de-gloving to prevent complications. In this paper we evaluate the feasibility of penile surgery avoiding circumcision. Materials and methods: patients presenting with penile curvature were treated using a modified Nesbit procedure. Patients were divided into group $A$ if they opted for a prepuce-sparing surgery and the others into group $B$. Patients were evaluated pre and postoperatively and postoperative complications were assessed. The 5-item International Index of Erectile Function (IIEF-5) was administered before and 6 months after surgery and we compared the difference of mean value using T-Test. Results: Group A and B were made of 53 and 16 patients respectively. Median age was 59 years [interquartile range (IQR) 12] in $A$ and 62 (IQR 9) in B $(p=0.2)$. Median curvature was $40^{\circ}\left(\right.$ IQR $\left.40^{\circ}\right)$ in $A$ and $40^{\circ}\left(\right.$ IQR $\left.30^{\circ}\right)$ in $B$ $(p=0.62)$. Mean difference between pre-and post-operative IEFF was $1.9 \pm 2$ in $A$ and $2.6 \pm 2.1$ in $B(p=0.36)$.

Conclusions: According to our experience, surgical correction of penile curvature without performing circumcision could be a safe and feasible strategy. We recommend performing circumcision only in patients who present with pre-operative phimosis.
\end{abstract}

KEY WORDS: Erectile dysfunction; Foreskin; Male; Phimosis; Penile induration.

Submitted 23 February 2020; Accepted 2 April 2020

\section{INTRODUCTION}

Penile curvature can be considered a relatively uncommon condition, with an incidence of 0,4-0,6\%. Estimated prevalence is about $0.5 \%$ but according to several Authors it may reach up to $10 \%$ due to misdiagnosis and low awareness of the disease $(1,2)$. While new promising therapies are developing and spreading (3-4), surgery is still indicated as primary management for many patients with penile curvatures, especially when the curvature does not allow a satisfactory intercourse (5-7). A common feature of the many different techniques of corporoplasty which were proposed over the last decades is to associate circumcision with penile degloving in order to prevent postoperative oedema, phimosis and necrosis of the prepuce $(8,9)$. However, few recent studies have shown that a prepuce-sparing surgery is feasible with a low rate of local complications $(10,11)$. We aim to evaluate the fea- sibility and the safety of prepuce-sparing corporoplasty on a single-institution series.

\section{MATERIALS AND MEthods}

Data were collected from patients presenting with Peyronie's disease (PD) who underwent to a modified Nesbit procedure from January 2014 to January 2019 at a single academic tertiary hospital. Our Institution's prospectively updated database that have been retrospectively evaluated. All the procedures were performed by the same experienced surgeon (A.F.D.R.).

\section{Preoperative assessment and follow-up}

All patients had a stable disease for at least three months and suffered from pain during erection or during sexual intercourse. All patients underwent a standard clinical assessment, routine blood sample, urinalysis, physical examination during erection (after intracavernous injection of vasoactive drugs or self-photography at orthogonal, frontal, and sagittal planes). Curvature degrees were measured using a goniometer. Both the prepuce and the degree of penile curvature were assessed before surgery. We considered curvature of more than $30^{\circ}$. Medical and sexual history of patients was taken focusing on any previous penile surgery. The 5-item International Index of Erectile Function (IIEF-5) was administered before and 6 months after the treatment. Degree of angulation was recorded at follow-up with self-photograph at orthogonal, frontal and sagittal planes.

\section{Sample definition and analysis}

The patients were divided into two different groups: group A, made by patients who opted for a prepucesparing surgery, and group B made by those who decided to be circumcised. Patients' characteristics, intra-, post-operative and 6 months follow-up data were collected and analysed. Post-operative complications were assessed according to Clavien-Dindo (CD) classification (14). In the two groups, we assessed the difference of mean value of IIEF-5 score administered before and 6 months after the surgery by using T-Test. Data were entered into a Microsoft Excel (Version 14.0) database and then transferred to Sofastat TM 1.4.6 for Windows. Descriptive statistics were calculated for all patients' variables and reported as median (IQR), mean \pm stan- 
dard deviation (SD) or as proportion. The homogeneity and data of the two groups were compared using the Kolmogorov-Smirnov, Chi-Square, Fisher Exact and the paired T-test. We set the level of significance to 0.05.

\section{Surgical procedure and perioperative management}

In all cases a modified Nesbit technique has been performed. The procedure starts with a sub-coronal circumferential incision $1 \mathrm{~cm}$ below the coronal sulcus. Then the degloving is carried out following the avascular plane between the dartos and the Buck's fascia. We accurately spare the dartos in order not to damage the blood supply to the prepuce. The neurovascular bundle is mobilized from the dorsum of the penis by incising Buck's fascia laterally. Five micro-grams of prostaglandin E1 (PGE 1) are injected into the corpora cavernosa to obtain an artificial erection.

The tunica albuginea can be grasped with an Allis clamp or with suspension stitches and then it is excised sharply with a cold scalpel (Figure 1).

The albuginea continuous solution is closed with interrupted polyglycolic 3/0 suture. The result is assessed by inducing a final hydraulic erection. Buck's fascia is reapproximated with an absorbable monofilament suture. Finally, we perform an interrupted 4/0 suture to approximate the skin (Figure 2).

For patients among group B the surgical procedure was similar to the technique performed for group A except for the final step of the circumcision. The redundant prepuce is pulled upwards, the adhesions to the glans is released, and after skin excision, haemostasis is performed with an electrocautery. We approximate the skin and the mucosal borders with simple interrupted absorbable suture (polyglycolic 3/0).

A transurethral catheter is placed during surgery and left for 1-2 days. All patients were premedicated with third

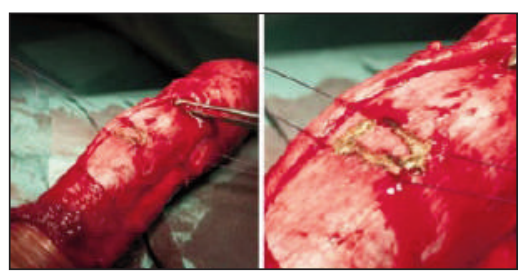

Figure 1

The electric scalpel delimits the area of corporoplasty subsequently engraved with the cold knife.

\section{Figure 2.}

Sub-coronal suture with Vycril rapid 4/0 stitches without circumcision.

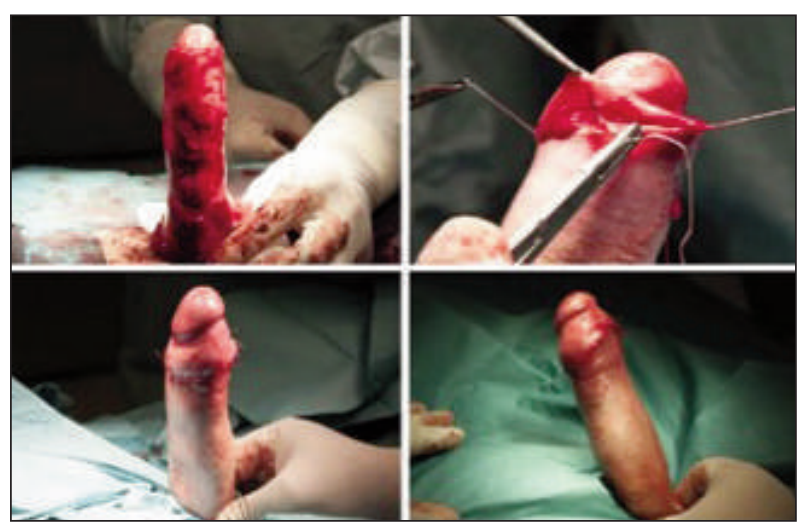

generation cephalosporins as prophylactic antibiotic before induction of anaesthesia $(12,13)$.

\section{Ethics statement}

Our institution doesn't require an Institutional review board approval for retrospective observational studies. However, every patient at the admission had to give a written consent for the prospective collection and retrospective analysis of data.

\section{ResUlts}

Group A and B were made of 53 and 16 patients, respectively. The median age was 59 years (IQR 12) in group A and $62(\mathrm{IQR} 9)$ in group $\mathrm{B}(\mathrm{p}=0.20)$. Median grade of penile curvature was $40^{\circ}$ (IQR $40^{\circ}$ ) in group A and $40^{\circ}$ $\left(\mathrm{IQR} 30^{\circ}\right)$ in group $\mathrm{B}(\mathrm{p}=0.62)$. The two groups were similar in direction of curvature (Table 1). Median operating time was 90 minutes (IQR 6) in group A and 100 minutes (IQR 30) in group B $(p=0.03)$. Patients of both two groups had their urethral catheters removed at a median of 1 day (IQR group A 1 and IQR group B 0) after surgery $(p=0.23)$. Median length of stay was 2 day (IQR 1) in group A and 2 day (IQR 0) in group B. Twenty-one patients of group A were used to take phosphodiesterase type 5 inhibitor (PDE5 inhibitor) before surgery. After the treatment 19 (36\%) of them continued to use PDE5 inhibitor while two of them started using intracavernous injections of vasoactive medication.

Table 1.

Summary table on the population of patients considered in the study.

\begin{tabular}{|lccc|}
\hline Variable & Group A & Group B & P value \\
\hline Number of patients & 53 & 16 & 0.91 \\
\hline Agge $(y)$, median $(\text { IQR })^{\text {a }}$ & $59(12)$ & $62(9)$ & 0.20 \\
\hline Curvature $\left({ }^{\circ}\right)$, median $($ IQR $)$ & $40^{\circ}\left(40^{\circ}\right)$ & $40^{\circ}\left(30^{\circ}\right)$ & 0.62 \\
\hline Direction of curvature, $n(\%)$ & $18(34)$ & $7(44)$ & 0.74 \\
\hline Dorsal & $16(30)$ & $5(31)$ & 0.98 \\
\hline Ventral & $19(36)$ & $4(25)$ & 0.59 \\
\hline Lateral & $19 \pm 2$ & $17 \pm 2$ & 0.02 \\
\hline IEFF-5 score, mean $\pm S D$ & & & \\
\hline a IQR $=$ interquartile range. & & & \\
\hline
\end{tabular}

Table 2.

Summary table on the population of patients considered in the study.

\begin{tabular}{|lccc|}
\hline Variable & Group A & Group B & P value \\
\hline Operating time (minutes), median (IQR) & $90(6)$ & $100(30)$ & 0.03 \\
\hline Duration of catheterization (days), median (IQR) & $1(1)$ & $1(0)$ & 0.23 \\
\hline Hospital stay (d), median (IQR) & $1(1)$ & $1(0)$ & 0.24 \\
\hline Complications & & & \\
CD ${ }^{\text {G Grade III }}$ & 4 & 0 & \\
CD Grade IV & 0 & 0 & \\
\hline IIEF-5 score 6 months after, mean \pm SD & $21 \pm 2$ & $20 \pm 2$ & 0.11 \\
\hline IIEF-5 pre-/post-op. difference & $1.9 \pm 2$ & $2.6 \pm 2.1$ & 0.36 \\
\hline Residual curvature at 6 months & 51 & 16 & \\
Straight & 2 & 0 & \\
$<15^{\circ}$ & & & \\
\hline${ }^{\circ} \mathrm{CD}=$ Clavien Dindo. & & \\
\hline
\end{tabular}


In group B, three patients used PDE5 inhibitor before surgery and they continued the same therapy also after the surgery. Mean difference between pre- and postoperative IIEF was $1.9 \pm 2$ in group A and $2.6 \pm 2.1$ in group B $(p=0.36)$. Four patients $(7.5 \%)$ among Group A experienced CD grade III postoperative complications (secondary circumcision due to post-operative paraphimosis/edema). No CD grade IV complications were reported in both groups. Two patients among group A reported recurrent curvature of more than 15 degrees, but in both cases no secondary surgery was required (Table 2).

\section{Discussion}

Penile curvature is a congenital or acquired deformity which results in sexually debilitating condition and significant psychological stress for patients and their partners (15). Once medical treatment is excluded or unuseful, surgery becomes the standard of choice (16).

Both shortening and lengthening procedures are good options for patients. The first technique to correct penile curvature was described by Nesbit (17). In the following years various modifications to Nesbit's technique were suggested such as the Yachia corporoplasty technique with or without penile degloving. According to the literature the outcomes of the "degloving" and "without degloving" techniques are similar (18).

Regardless of the procedure performed, penile degloving is generally combined with circumcision to reduce risk of postoperative oedema of the prepuce, with subsequent phimosis and necrosis $(19,20)$. However, recent studies demonstrate that circumcision is not always necessary, particularly when patients present with no phimosis pre-operatively $(10,11)$. Alei et al. (21) described a new corporoplasty technique named as the doublebreasted' corporoplasty, with penoscrotal and infrapubic access not requiring circumcision. They reported no major complications nor neurovascular lesions or change in erectile function, low morbidity, low recurrence rate and excellent aesthetic results. Garaffa et al. (10) reviewed a series of 251 patients who underwent a Lue (86 patients) or a Nesbit procedure (162 patients) or a combination of both. They reported very few complications in the group of patients without pre-operatively phimosis that chose not to be circumcised. They could state that circumcision should not be considered as a routine part of penile surgery unless a significant phimosis is present. Similarly, Pavone et al. (11) reported a series of 147 patients treated with Nesbit's corporoplasty without circumcision and no major complication were described. The authors affirmed that circumcision must not be considered a mandatory time in Nesbit procedure if the anatomical structures are carefully respected, the number of artificial erections is reduced.

In this paper we analysed surgical outcomes of a group of patients treated with modified Nesbit procedure without circumcision comparing them with a similar group treated with the same technique but completed with circumcision. Pre and post-operative data were similar in both two groups and no major complications were reported.

Not performing circumcision in penile surgery could be a safe strategy if some precautions are followed. We recommend minimizing trauma of tissues carefully isolating the plane between the dartos and the Buck's fascia to avoid bleeding and ischaemia. In addition, the initial subcoronal circumferential incision $1 \mathrm{~cm}$ below the coronal sulcus allows to spare a sufficient prepuce vascularization.

In this way the risk of tissue retraction and consequently to phimosis and foreskin necrosis could be reduced. Overall, regardless of prepuce sparing surgery, reported success rate with tunica albuginea plication procedures is about $85 \%$, with a good erectile function (IIEF- $5>21$ in more than $85 \%$ of patients) (22).

In our analysis surgical correction of penile curvature has a good impact on erectile function (IIEF score improve in both two groups) and the comparison of the IIEF difference between the 2 groups didn't show any statistically significant difference.

Thus, it seems that if circumcision is not performed sexual outcomes could not be negatively affected.

Many limitations exist in the current research beginning with the study design because of the retrospective nature of the study and the limited sample. However, the Institution's database has been prospectively updated and the size of the sample, even if small, is comparable to that of other previous studies. Furthermore, the enrolment period is quite long.

Anyway, to the best of our knowledge, the literature on this topic is weak and we couldn't find any study on this subject with a prospective design.

\section{Conclusions}

In conclusion, according to our experience, surgical correction of penile curvature without performing circumcision could be a feasible and safety strategy with low rate of foreskin complications. Additional prospective studies with larger patient cohorts are required to draw stronger conclusion.

\section{Aknowledgments}

The authors acknowledge the Clinical risk management, quality, accreditation and public relation Unit of their Hospital that actively participated to the development of the research.

\section{REFERENCES}

1. Yachia D, Beyar M, Aridogan IA, Dascalu S. The incidence of congenital penile curvature. J Urol. 1993; 150:1478-9.

2. Stuntz M, Perlaky A, Des Vignes F, et al. The prevalence of Peyronie's disease in the United States: A population-based study. PLoS One. 2016; 11:e0150157.

3. Liu T, Shindel AW, Lin G, Lue TF. Cellular signaling pathways modulated by low-intensity extracorporeal shock wave therapy. Int J Impot Res. 2019; 31:170-176.

4. Cocci A, Cito G, Urzi D, et al. Sildenafil $25 \mathrm{mg} O D T+$ Collagenase Clostridium hystoliticum vs Collagenase Clostridium hystoliticum alone for the management of Peyronie's disease: a matched-pair comparison analysis. J Sex Med. 2018; 15:1472-1477. 
5. García-Gómez B, González-Padilla DA, Alonso-Isa M, et al. Plication techniques in Peyronie's disease: new developments Int $J$ Impot Res. 2020; 32:30-36.

6. Cosentino M, Kanashiro A, Vives A, et al. Surgical treatment of Peyronie's disease with small intestinal submucosa graft patch. Int J Impot Res. 2016; 28:106-109.

7. Hatzimouratidis K, Giuliano F, Moncada I, et al. EAU Guidelines on Erectile Dysfunction, Premature Ejaculation, Penile Curvature and Priapism. www.uroweb.org 2019.

8. Nyirady P, Kelemen Z, Banfi G, et al. Management of congenital penile curvature. J Urol. 2008; 179:1495-8.

9. Langston JP, Carson CC $3^{\text {rd }}$. Peyronie disease: plication or grafting. Urol Clin North Am. 2011; 38:207-16.

10. Garaffa G, Sacca A, Christopher AN, Ralph DJ. Circumcision is not mandatory in penile surgery. BJU Int. 2010; 105:222-4.

11. Pavone C, Abbadessa D, Usala M, et al. Circumcision in Nesbit corporoplasty: a mandatory time? Our experience on 158 patients. Urologia. 2012; 79:44-8.

12. Van Der Horst C, Martinez Portillo FJ, Seif C, et al. Treatment of penile curvature with Essed-Schroder tunical plication: aspects of quality of life from the patients' perspective. BJU Int. 2004; 93:105-8.

13. Mantovani F, Patelli E, Castelnuovo C, Nicola M. 'Straighteningreinforcing' technique for congenital curvature and Peyronie's disease. Urol Int. 2005; 75:201-3.

14. Clavien PA, Barkun J, de Oliveira ML, et al. The Clavien-Dindo classification of surgical complications: five-year experience. Ann Surg. 2009; 250:187-96.

15. De Rose AF, Mantica G, Maffezzini M, Terrone C. Dermal graft surgery for Peyronie's disease: Long term results at a 15 years follow-up. Arch Esp Urol. 2019; 72:415-421.

16. Sokolakis I, Schönbauer P, Mykoniatis I, et al. Long-term results after surgical treatment of congenital penile curvature using a modified Nesbit technique World J Mens Health. 2019; 4 [Online ahead of print]

17. Nesbit RM. Congenital curvature of the phallus: report of three cases with description of corrective operation. J Urol. 1965; 171:230-232.

18. Dell'Atti L, Polito M, Galosi A. B. Is degloving the best method to approach the penile corporoplasty with Yachia's technique? Urology. 2019; 126:204-208.

19. Kalsi JS, Christopher N, Ralph DJ, Minhas S. Plaque incision and fascia lata grafting in the surgical management of Peyronie's disease. BJU Int 2006; 98:110-510.

20. Adeniyi AA, Goorney SR, Pryor JP, Ralph DJ. The Lue procedure: an analysis of the outcome in Peyronie's disease. BJU Int. 2002; 89:404-8.

21. Alei $G$, Letizia P, Alei $L$, et al. New surgical technique for ventral penile curvature without circumcision. BJU Int. 2014; 113:968-74.

22. Savoca G, Scieri F, Pietropaolo F, et al. Straightening corporoplasty for Peyronie's disease: a review of 218 patients with median follow-up of 89 months. Eur Urol. 2004; 46: 610-4.

\section{Correspondence}

Aldo Franco De Rose, MD

aldofdr@libero.it

Francesca Ambrosini, MD (Corresponding Author)

f.ambrosinil@gmail.com

ORCID 0000-0003-2160-763X

Guglielmo Mantica, MD

guglielmo.mantica@gmail.com

Riccardo Banchero, MD

riccardo.banchero@hsanmartino.it

Carlo Terrone, $M D$

carlo.terrone@med.uniupo.it

Department of Urology, Policlinico San Martino Hospital, University of Genoa

Largo Rosanna Benzi, 10, 16132, Genoa, Italy

Enrico Zero, $M D$

zero.enrico@gmail.com

Department of Computer Science, Bioengineering, Robotics and Systems

Engineering, University of Genoa, Genoa, Italy 